\title{
Gene microarray analyses for potential biomarkers of single and recurrent venous thromboembolism
}

\author{
WUGANG ZHOU ${ }^{1}$, KE ZHANG ${ }^{2}$, DONGRUI CHEN ${ }^{2}$, PINGJIN GAO $^{2}$ and QIAO WANG ${ }^{1}$ \\ ${ }^{1}$ Emergency Department, Shanghai Ninth People's Hospital, Shanghai Jiaotong University School of Medicine, \\ Shanghai 200011; ${ }^{2}$ Department of Hypertension, Ruijin Hospital, Shanghai Key Lab of Hypertension, \\ Shanghai Institute of Hypertension, Shanghai Jiaotong University School of Medicine, Shanghai 200025, P.R. China
}

Received November 13, 2014; Accepted August 4, 2015

DOI: $10.3892 / \mathrm{mmr} .2015 .4349$

\begin{abstract}
Venous thromboembolism is a major cause of morbidity and mortality with a high recurrence rate. The present study aimed to explore the molecular mechanisms and potential biomarkers of single venous thromboembolism (SVTE) and recurrent venous thromboembolism (RVTE). The microarray dataset GSE19151 was downloaded from Gene Expression Omnibus, which contained data from whole blood samples from 63 healthy controls, 32 SVTE and 38 RVTE patients. Differentially expressed genes (DEGs) in the SVTE and RVTE groups compared with those in the controls were identified using the $t$-test, followed by clustering analysis of DEGs and samples. Functional and pathway enrichment analyses were performed for DEGs in patients with RVTE and SVTE, as well as specific DEGs in patients with RVTE. The identified 42 DEGs in RVTE were mainly enriched in biological processes of cellular protein metabolism, gene expression and translational elongation as well as in pathways associated with ribosomes, Parkinson's disease and oxidative phosphorylation. In SVTE, 20 DEGs were identified, which were mainly involved in biological processes of biopolymer biosynthesis, translational elongation and cellular protein metabolism as well as pathways associated with ribosomes and cardiac muscle contraction. In RVTE, 22 specific DEGs were mainly involved in translational elongation, negative regulation of the force of heart contraction by chemical signals, cell proliferation, ribosomal pathways and protein export. The identified DEGs of SVTE, including COX7C and UQCRQ, may be potential biomarkers for SVTE, and the specific DEGs of RVTE, including ADRBK1, NDUFA5 and ATP5O, may be potential biomarkers for RVTE.
\end{abstract}

Correspondence to: Dr Qiao Wang, Emergency Department, Shanghai Ninth People's Hospital, Shanghai Jiaotong University School of Medicine, 639 Zhizaoju Road, Shanghai 200011, P.R. China

E-mail: qiaowangqw@163.com

Key words: venous thromboembolism, recurrence, biomarkers, bioinformatics

\section{Introduction}

Venous thromboembolism (VTE), comprising deep venous thrombosis and pulmonary embolism, is a frequent disease with high morbidity and mortality, which affects 1-2 per 1,000 individuals (1-3). Furthermore, VTE is associated with a significant rate of recurrence in as many as $30 \%$ of VTE patients after termination of the standard course of anti-coagulant therapy (4-6). Therefore, it is desired to explore the molecular mechanisms and potential biomarkers that enable clinicians to identify patients at a risk of single VTE (SVTE) or recurrent VTE (RVTE) for prompt clinical diagnosis and early prevention (7).

Kyrle et al (8) reported that patients with a high level of factor VIII have an increased risk of RVTE. A study by Comp and Esmon (9) suggested that the levels of protein $\mathrm{S}$ may be used in the evaluation of RVTE. Various established and novel biomarkers, including D-dimer, E-selectin, P-selectin, thrombin, inflammatory markers and C-reactive protein, have been investigated for their predictive value in SVTE and RVTE (10-13). However, only a small number of biomarkers, such as D-dimer, associated with a first or recurrent event of VTE were highlighted by these studies, while novel and promising biomarkers, including P-selectin and inflammatory cytokines, are still controversial (1). A study by Lewis et al (14) performed a pathway enrichment analysis of differentially expressed genes (DEGs) in samples from patients with SVTE and samples from patients with RVTE and found that insulin-like growth factor receptor 1 and Akt pathways may be useful for distinguishing patients with SVTE from those with RVTE.

The present study identified DEGs in RVTE and SVTE, as well as specific DEGs in RVTE. Functional and pathway enrichment analyses for these DEGs were performed to explore the molecular mechanisms and potential biomarkers of SVTE and RVTE in order to facilitate the diagnosis and clinical therapy management of VTE.

\section{Materials and methods}

Affymetrix microarray data. The gene expression profile dataset GSE19151 was obtained from Gene Expression Omnibus (http://www.ncbi.nlm.nih.gov/geo/), which was deposited by Lewis et al (14). Microarray data from 133 whole 
Table I. Specific differentially expressed genes of recurrent VTE.

\begin{tabular}{|c|c|c|c|c|c|c|}
\hline Probe ID & $\begin{array}{c}\text { Mean of recurrent } \\
\text { VTE (R) }\end{array}$ & $\begin{array}{c}\text { Mean of } \\
\text { healthy }(\mathrm{N})\end{array}$ & $\log 2(\mathrm{R} / \mathrm{N})$ & P-value & $\begin{array}{l}\text { Gene } \\
\text { symbol }\end{array}$ & $\begin{array}{l}\text { ENTREZ } \\
\text { GENE_ID }\end{array}$ \\
\hline 201304_at & 6.22 & 4.64 & 1.58 & $2.55 \times 10^{-5}$ & NDUFA5 & 4698 \\
\hline 200679_x_at & 9.86 & 8.67 & 1.19 & $4.85 \times 10^{-10}$ & HMGB1 & 3146 \\
\hline 201317_s_at & 9.49 & 8.16 & 1.33 & $2.18 \times 10^{-7}$ & PSMA2 & 5683 \\
\hline 200977_s_at & 9.41 & 8.16 & 1.24 & $1.39 \times 10^{-5}$ & TAX1BP1 & 8887 \\
\hline 201152_s_at & 11.86 & 10.69 & 1.17 & $1.17 \times 10^{-6}$ & MBNL1 & 4154 \\
\hline 201163_s_at & 8.79 & 7.54 & 1.24 & $1.19 \times 10^{-12}$ & IGFBP7 & 3490 \\
\hline 201593_s_at & 9.38 & 8.26 & 1.12 & $3.27 \times 10^{-10}$ & ZC3H15 & 55854 \\
\hline 201401_s_at & 8.57 & 9.69 & -1.13 & $1.39 \times 10^{-11}$ & ADRBK1 & 156 \\
\hline 200834_s_at & 12.54 & 11.54 & 1.01 & $1.97 \times 10^{-11}$ & RPS21 & 6227 \\
\hline 201367_s_at & 8.91 & 10.14 & -1.24 & $2.12 \times 10^{-7}$ & ZFP36L2 & 678 \\
\hline 201392_s_at & 9.09 & 10.22 & -1.13 & $5.39 \times 10^{-10}$ & IGF2R & 3482 \\
\hline 201200_at & 10.98 & 9.82 & 1.16 & $1.72 \times 10^{-11}$ & CREG1 & 8804 \\
\hline 200012_x_at & 13.08 & 11.66 & 1.42 & $1.40 \times 10^{-6}$ & RPL21 & 6144 \\
\hline 200061_s_at & 11.34 & 9.94 & 1.40 & $4.02 \times 10^{-9}$ & RPS24 & 6229 \\
\hline 200741_s_at & 13.56 & 12.34 & 1.22 & $1.28 \times 10^{-11}$ & RPS27 & 6232 \\
\hline 200880_at & 8.92 & 7.73 & 1.19 & $1.17 \times 10^{-7}$ & DNAJA1 & 3301 \\
\hline 201012_at & 10.84 & 9.29 & 1.55 & $2.33 \times 10^{-9}$ & ANXA1 & 301 \\
\hline 201273_s_at & 10.76 & 9.69 & 1.07 & 0.000332 & SRP9 & 6726 \\
\hline 201332_s_at & 8.22 & 9.35 & -1.13 & $1.44 \times 10^{-13}$ & STAT6 & 6778 \\
\hline 201406_at & 10.35 & 9.03 & 1.32 & $6.59 \times 10^{-5}$ & RPL36A & 6173 \\
\hline 201023_at & 9.93 & 8.76 & 1.17 & 0.000185 & TAF7 & 6879 \\
\hline 200818_at & 10.17 & 9.16 & 1.02 & $5.51 \times 10^{-5}$ & ATP5O & 539 \\
\hline
\end{tabular}

VTE, venous thromboembolism; R, recurrent; N, normal.

blood specimens were available, including 63 samples from healthy controls, 32 samples from patients with SVTE (sampled at $<$ three years since their most recent VTE) and 38 samples from subjects with recurrent venous thromboembolism (RVTE) on warfarin. The platform was GPL571 (HG-U133A_2) Affymetrix Human Genome U133A 2.0 Array (Affymetrix, Inc., Santa Clara, CA, USA).

DEG analysis and gene clustering analysis. For the GSE19151 dataset, the Bioconductor software package in R (http://www. bioconductor.org/; version 3.1) was implemented to analyze the 133 blood gene chips (15). Background correction and quartile data normalization were performed using the robust multiarray average algorithm with defaulted parameters in the Affy package (http://www.bioconductor.org/packages/release/ bioc/html/affy.html; version 1.46.1) (16). The t-test was used to identify DEGs using the Simpleaffy package (http://www. bioconductor.org/packages/release/bioc/html/simpleaffy.html; version 2.44.0) (17). The DEGs were selected with the cutoff criteria of $\mathrm{P}<0.05$ and $\mid \log$ (fold change) $\mid>1$. Hierarchical clustering analysis of the DEGs was performed using the Hclust command in $\mathrm{R}$ and the default complete linkage method (18).

Gene ontology (GO) functional and pathway enrichment analyses. The Integrated GEne and PROtein annotation Server (IGEPROS; http://www.biosino.org/iGepros/index. jsp) (19) bioinformatics resources consist of an integrated biological knowledge base and analytic tools aimed at systematically extracting biological information from large gene or protein lists. IGEPROS was used to perform the GO (http:// www.geneontology.org/) functional and Kyoto Encyclopedia of Genes and Genomes (KEGG; http://www.genome.jp/kegg/ pathway.html) pathway enrichment analyses for the identified DEGs with the threshold of $\mathrm{P}<0.05$. The pathview package in $\mathrm{R}$ was utilized to depict the KEGG pathway (20).

\section{Results}

DEG selection and hierarchical clustering analysis. A total of 42 DEGs were identified between RVTE and normal whole-blood specimens (RVTE vs. control), including 35 upand 7 downregulated genes. Subsequently, 20 DEGs between SVTE and normal whole-blood specimens (SVTE vs. control) were identified, including 17 up- and 3 downregulated genes. A total of 22 non-overlapping genes were selected as specific DEGs of RVTE, including 18 up- and 4 downregulated genes (Table I). Hierarchical clustering analysis was performed for the 42 DEGs from the 133 whole blood specimens of patients with SVTE, patients with RVTE and healthy controls. The result of this clustering analysis suggested that these DEGs may have important roles in VTE (Fig. 1).

GO enrichment analysis of DEGs. GO enrichment analysis was performed for 42 DEGs in RVTE, 20 DEGs in SVTE 

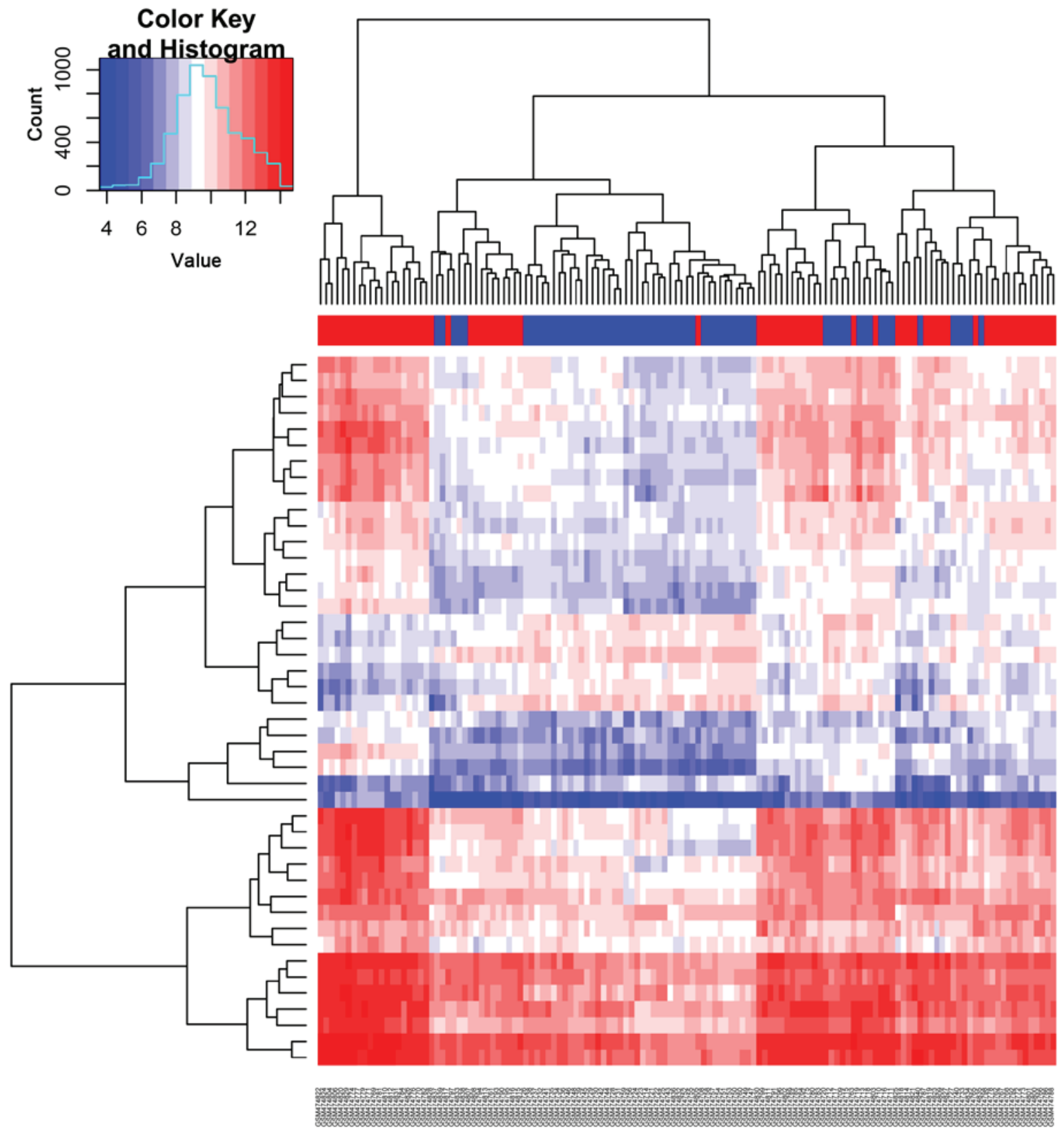

Figure 1. Clustering dendrogram constructed using the Hclust clustering algorithm. Horizontal red and blue bars represent the patient specimens (single or recurrent venous thromboembolism) and healthy specimens, respectively, and vertical axes represent differentially expressed genes. Expression levels are represented by a color key in which bright red represents the lowest levels and bright yellow represents the highest levels, and less saturated shades represent intermediate levels of expression.

and 22 specific DEGs of RVTE. In RVTE, most enriched $\mathrm{GO}$ terms of DEGs in biological processes were associated with biopolymer biosynthesis, including cellular protein metabolism $\left(\mathrm{P}=1.12 \times 10^{-8}\right)$, gene expression $\left(\mathrm{P}=1.36 \times 10^{-6}\right)$, translational elongation $\left(\mathrm{P}=9.65 \times 10^{-27}\right)$ and cellular macromolecular biosynthetic processes $\left(\mathrm{P}=8.78 \times 10^{-6}\right)$. In the cellular component category, enriched GO terms were mainly associated with the ribosomal sub-unit $\left(\mathrm{P}=2.03 \times 10^{-18}\right)$, cytosol $\left(\mathrm{P}=2.43 \times 10^{-12}\right)$ and macromolecular complexes $\left(\mathrm{P}=8.61 \times 10^{-11}\right)$. In the molecular function category, GO terms enriched for DEGs in RVTE included structural constituents of ribosomes $\left(\mathrm{P}=9.13 \times 10^{-24}\right)$, insulin-like growth factor binding $(\mathrm{P}=0.002)$ and beta-adrenergic receptor kinase activity $(\mathrm{P}=0.005)$ (Tables II-IV).
In SVTE, the most significantly enriched GO terms for DEGs in biological processes included biopolymer biosynthesis $\left(\mathrm{P}=2.35 \times 10^{-5}\right)$, translational elongation $\left(\mathrm{P}=2.35 \times 10^{-5}\right)$, cellular protein metabolism $\left(\mathrm{P}=1.24 \times 10^{-7}\right)$ and rRNA processing $(\mathrm{P}=0.006)$. The predominantly enriched GO terms of the cellular component category were the cytosol $\left(\mathrm{P}=1.68 \times 10^{-11}\right)$, the cytosolic large ribosomal sub-unit $\left(\mathrm{P}=8.62 \times 10^{-10}\right)$ and macromolecular complexes $\left(\mathrm{P}=4.86 \times 10^{-9}\right)$. The main $\mathrm{GO}$ terms of DEGs in SVTE in the molecular function category were structural constituents of ribosomes $\left(\mathrm{P}=4.69 \times 10^{-20}\right)$, RNA binding $\left(\mathrm{P}=2.79 \times 10^{-5}\right)$ and protein channel activity $(\mathrm{P}=0.001)$. Among the DEGs in SVTE, 12 genes, including RPS3A and RPS7, were involved in translational elongation, 2 genes (UQCRQ and COX7C) were involved in oxidoreductase 
Table II. GO functional enrichment analysis of DEGs in patients with RVTE and SVTE, and top 10 specific DEGs of RVTE associated with biological processes.

\begin{tabular}{|c|c|c|c|c|}
\hline Category/GOBPID & P-value & Count (n) & Size (n) & Term \\
\hline \multicolumn{5}{|l|}{ RVTE } \\
\hline GO:0006414 & $9.65 \times 10^{-27}$ & 17 & 104 & Translational elongation \\
\hline GO:0044267 & $1.12 \times 10^{-8}$ & 22 & 2361 & Cellular protein metabolic process \\
\hline GO:0010467 & $1.36 \times 10^{-6}$ & 25 & 3592 & Gene expression \\
\hline GO:0043284 & $2.88 \times 10^{-6}$ & 23 & 3183 & Biopolymer biosynthetic process \\
\hline GO:0034645 & $8.78 \times 10^{-6}$ & 23 & 3386 & Cellular macromolecule biosynthetic process \\
\hline GO:0044237 & $1.49 \times 10^{-5}$ & 34 & 7160 & Cellular metabolic process \\
\hline GO:0009058 & 0.000114 & 24 & 4223 & Biosynthetic process \\
\hline GO:0042274 & 0.000360 & 2 & 10 & Ribosomal small subunit biogenesis \\
\hline GO:0044238 & 0.001536 & 31 & 7368 & Primary metabolic process \\
\hline GO:0006364 & 0.002059 & 3 & 88 & rRNA processing \\
\hline \multicolumn{5}{|l|}{ SVTE } \\
\hline GO:0006414 & $7.47 \times 10^{-22}$ & 12 & 106 & Translational elongation \\
\hline GO:0044267 & $1.24 \times 10^{-7}$ & 14 & 2499 & Cellular protein metabolic process \\
\hline GO:0043284 & $2.35 \times 10^{-5}$ & 13 & 3183 & Biopolymer biosynthetic process \\
\hline GO:0034645 & $4.76 \times 10^{-5}$ & 13 & 3386 & Cellular macromolecule biosynthetic process \\
\hline GO:0010467 & $9.42 \times 10^{-5}$ & 11 & 3052 & Gene expression \\
\hline GO:0044237 & 0.000402 & 17 & 7160 & Cellular metabolic process \\
\hline GO:0009058 & 0.000549 & 13 & 4223 & Biosynthetic process \\
\hline GO:0006610 & 0.004003 & 1 & 3 & Ribosomal protein import into nucleus \\
\hline GO:0006364 & 0.006046 & 2 & 88 & rRNA processing \\
\hline GO:0042273 & 0.010641 & 1 & 8 & Ribosomal large subunit biogenesis \\
\hline \multicolumn{5}{|l|}{ Non-overlap } \\
\hline GO:0006414 & $3.52 \times 10^{-7}$ & 5 & 104 & Translational elongation \\
\hline GO:0000296 & 0.001547 & 1 & 1 & Spermine transport \\
\hline GO:0003108 & 0.001547 & 1 & 1 & $\begin{array}{l}\text { Negative regulation of the force of heart contraction by } \\
\text { chemical signal }\end{array}$ \\
\hline GO:0008283 & 0.002157 & 6 & 917 & Cell proliferation \\
\hline GO:0044267 & 0.002941 & 9 & 2361 & Cellular protein metabolic process \\
\hline GO:0006916 & 0.002998 & 3 & 190 & Anti-apoptosis \\
\hline GO:0048523 & 0.003033 & 7 & 1334 & Negative regulation of cellular process \\
\hline GO:0045988 & 0.003092 & 1 & 2 & Negative regulation of striated muscle contraction \\
\hline GO:0045900 & 0.003092 & 1 & 2 & Negative regulation of translational elongation \\
\hline GO:0048295 & 0.003092 & 1 & 2 & Positive regulation of isotype switching to IgE isotypes \\
\hline
\end{tabular}

GO, gene ontology; DEG, differentially expressed genes; RVTE, recurrent venous thromboembolism; SVTE, single venous thromboembolism; non-overlap, non-overlapping DEGs as specific DEGs of RVTE; GOBPID, gene ontology biological process identification number; IgE, immunoglobulin E; Count, DEG numbers enriched in the specific GO term; Size, total gene numbers in the GO database.

activity and 2 genes (HINT1 and LAMP1) were associated with proteolysis (Tables II-IV).

The GO terms for biological processes of the 22 specific DEGs of RVTE were mainly translational elongation $\left(\mathrm{P}=3.52 \times 10^{-7}\right)$, negative regulation of the force of heart contraction by chemical signals $(\mathrm{P}=0.001)$ and cell proliferation $(\mathrm{P}=0.002)$. In the cellular component category, enriched GO terms were mainly associated with the cytosolic small ribosomal sub-unit $\left(\mathrm{P}=1.56 \times 10^{-5}\right)$, ribosomal sub-unit $\left(\mathrm{P}=1.84 \times 10^{-5}\right)$ and macromolecular complexes $(\mathrm{P}=0.0008)$. The specific $\mathrm{GO}$ terms of the 22 specific
DEGs in RVTE in the molecular function category were mainly associated with structural constituents of ribosomes $\left(\mathrm{P}=2.55 \times 10^{-6}\right)$, insulin-like growth factor binding $(\mathrm{P}=0.0006)$, beta-adrenergic receptor kinase activity $(\mathrm{P}=0.003)$ and phospholipase $\mathrm{A} 2$ inhibitor activity $(\mathrm{P}=0.004)$. Two genes (IGF2R and IGFBP7) were shown to be involved in anti-apoptotic signaling, ten genes, including RPL21 and RPS21, were involved in translational elongation and six genes, including NDUFA5, ATP5O and ADRBK1, were associated with the force of heart contraction (Tables II-IV). 
Table III. GO functional enrichment analysis of DEGs in patients with RVTE and SVTE, and top 10 specific DEGs of RVTE associated with cellular components.

\begin{tabular}{|c|c|c|c|c|}
\hline Category/GOCCID & P-value & Count (n) & Size $(n)$ & Term \\
\hline \multicolumn{5}{|l|}{ RVTE } \\
\hline GO:0033279 & $2.03 \times 10^{-18}$ & 13 & 118 & Ribosomal sub-unit \\
\hline GO:0005829 & $2.43 \times 10^{-12}$ & 19 & 1039 & Cytosol \\
\hline GO:0032991 & $8.61 \times 10^{-11}$ & 25 & 2482 & Macromolecular complex \\
\hline GO:0022627 & $4.11 \times 10^{-10}$ & 6 & 36 & Cytosolic small ribosomal sub-unit \\
\hline GO:0022625 & $5.80 \times 10^{-10}$ & 6 & 38 & Cytosolic large ribosomal sub-unit \\
\hline GO:0043228 & $2.18 \times 10^{-9}$ & 23 & 2388 & Non-membrane-bound organelle \\
\hline GO:0005737 & $1.12 \times 10^{-7}$ & 35 & 6946 & Cytoplasm \\
\hline GO:0005840 & $1.16 \times 10^{-5}$ & 4 & 78 & Ribosome \\
\hline GO:0005622 & $5.89 \times 10^{-5}$ & 39 & 10646 & Intracellular \\
\hline GO:0005730 & 0.001773 & 7 & 680 & Nucleolus \\
\hline \multicolumn{5}{|l|}{ SVTE } \\
\hline GO:0005829 & $1.68 \times 10^{-11}$ & 13 & 1039 & Cytosol \\
\hline GO:0022625 & $8.62 \times 10^{-10}$ & 5 & 38 & Cytosolic large ribosomal sub-unit \\
\hline GO:0032991 & $4.86 \times 10^{-9}$ & 15 & 2482 & Macromolecular complex \\
\hline GO:0043228 & $4.10 \times 10^{-8}$ & 14 & 2388 & Non-membrane-bound organelle \\
\hline GO:0044422 & $2.04 \times 10^{-6}$ & 15 & 3829 & Organelle part \\
\hline GO:0022627 & $1.16 \times 10^{-5}$ & 3 & 36 & Cytosolic small ribosomal sub-unit \\
\hline GO:0033279 & $1.53 \times 10^{-5}$ & 3 & 57 & Ribosomal sub-unit \\
\hline GO:0005840 & $1.82 \times 10^{-5}$ & 3 & 78 & Ribosome \\
\hline GO:0043229 & $7.82 \times 10^{-5}$ & 19 & 8645 & Intracellular organelle \\
\hline GO:0005622 & 0.000279 & 20 & 10646 & Intracellular \\
\hline \multicolumn{5}{|l|}{ Non-overlap } \\
\hline GO:0022627 & $1.56 \times 10^{-5}$ & 3 & 36 & Cytosolic small ribosomal sub-unit \\
\hline GO:0033279 & $1.84 \times 10^{-5}$ & 4 & 118 & Ribosomal sub-unit \\
\hline GO:0032991 & 0.000837 & 10 & 2482 & Macromolecular complex \\
\hline GO:0005737 & 0.001286 & 17 & 6946 & Cytoplasm \\
\hline GO:0008024 & 0.001373 & 1 & 1 & Positive transcription elongation factor complex $b$ \\
\hline GO:0005829 & 0.002221 & 6 & 1039 & Cytosol \\
\hline GO:0005785 & 0.002744 & 1 & 2 & Signal recognition particle receptor complex \\
\hline GO:0043232 & 0.002811 & 9 & 2388 & Intracellular non-membrane-bound organelle \\
\hline GO:0005641 & 0.006847 & 1 & 5 & Nuclear envelope lumen \\
\hline GO:0044422 & 0.006937 & 11 & 3829 & Organelle part \\
\hline
\end{tabular}

GO, gene ontology; DEG, differentially expressed genes; RVTE, recurrent venous thromboembolism; SVTE, single venous thromboembolism; Non-overlap, non-overlapping DEGs as specific DEGs of RVTE; GOCCID, gene ontology cellular component identification number; Count, DEG numbers enriched in the specific GO term; Size, total gene numbers in the GO database.

Pathway enrichment analysis of DEGs. The DEGs identified in the present study were enriched in nine pathways (Table V). The RVTE DEGs were mainly enriched in ribosomal pathways $\left(\mathrm{P}=1.59 \times 10^{-23}\right)$, Parkinson's disease $(\mathrm{P}=0.007)$ and oxidative phosphorylation $(\mathrm{P}=0.008)$. The SVTE DEGs were enriched in ribosomal pathways $\left(\mathrm{P}=4.58 \times 10^{-19}\right)$ and cardiac muscle contraction $(\mathrm{P}=0.025)$. The non-overlapping $\mathrm{DEGs}$ were enriched in ribosomal pathways $\left(\mathrm{P}=2.25 \times 10^{-6}\right)$ and protein export $(\mathrm{P}=0.03)$.

Metabolic pathway visualization analysis of specific DEGs of RVTE. The metabolic pathways were visualized in schemes depicting the ribosomal pathway (Fig. 2) and the protein export pathway (Fig. 3; http://www.genome.jp/kegg/tool/map_ pathway2.html). In the ribosomal pathway, RPL21, RPS21, RPS24 and RPS27 were upregulated and in the protein export pathway, SRP9 was upregulated. The results suggested that these genes may be critical in RVTE and that certain variations in the expression of these genes may lead to an increased risk of recurrence.

\section{Discussion}

In recent years, the application of adequate thromboprophylaxis has led to significant progress in the management of 
Table IV. GO functional enrichment analysis of DEGs in patients with RVTE and SVTE, and top 10 specific DEGs of RVTE associated with molecular function.

\begin{tabular}{|c|c|c|c|c|}
\hline Category/GOMFID & P-value & Count (n) & Size (n) & Term \\
\hline \multicolumn{5}{|l|}{ RVTE } \\
\hline GO:0003735 & $9.13 \times 10^{-24}$ & 17 & 158 & Structural constituent of ribosome \\
\hline GO:0003723 & $2.87 \times 10^{-6}$ & 10 & 646 & RNA binding \\
\hline GO:0005520 & 0.002031 & 2 & 25 & Insulin-like growth factor binding \\
\hline GO:0015266 & 0.002686 & 1 & 1 & Protein channel activity \\
\hline GO:0015077 & 0.002942 & 3 & 107 & Monovalent inorganic cation transmembrane transporter activity \\
\hline GO:0047696 & 0.005365 & 1 & 2 & Beta-adrenergic receptor kinase activity \\
\hline GO:0005010 & 0.008037 & 1 & 3 & Insulin-like growth factor receptor activity \\
\hline GO:0019834 & 0.008037 & 1 & 3 & Phospholipase A2 inhibitor activity \\
\hline GO:0003729 & 0.008574 & 2 & 52 & mRNA binding \\
\hline GO:0005047 & 0.010702 & 1 & 4 & Signal recognition particle binding \\
\hline \multicolumn{5}{|l|}{ SVTE } \\
\hline GO:0003735 & $4.69 \times 10^{-20}$ & 12 & 158 & Structural constituent of ribosome \\
\hline GO:0003723 & $2.79 \times 10^{-5}$ & 6 & 630 & RNA binding \\
\hline GO:0015266 & 0.001245 & 1 & 1 & Protein channel activity \\
\hline GO:0003729 & 0.001876 & 2 & 52 & mRNA binding \\
\hline GO:0015077 & 0.007702 & 2 & 107 & Monovalent inorganic cation transmembrane transporter activity \\
\hline GO:0008121 & 0.009917 & 1 & 8 & Ubiquinol-cytochrome $\mathrm{C}$ reductase activity \\
\hline GO:0016679 & 0.009917 & 1 & 8 & $\begin{array}{l}\text { Oxidoreductase activity, acting on diphenols and associated } \\
\text { substances as donors }\end{array}$ \\
\hline GO:0030552 & 0.017294 & 1 & 14 & cAMP binding \\
\hline GO:0005080 & 0.018518 & 1 & 15 & Protein kinase $\mathrm{C}$ binding \\
\hline GO:0008603 & 0.019741 & 1 & 16 & cAMP-dependent protein kinase regulator activity \\
\hline \multicolumn{5}{|l|}{ Non-overlap } \\
\hline GO:0003735 & $2.55 \times 10^{-6}$ & 5 & 158 & Structural constituent of ribosome \\
\hline GO:0005520 & 0.000583 & 2 & 25 & Insulin-like growth factor binding \\
\hline GO:0047696 & 0.002881 & 1 & 2 & Beta-adrenergic receptor kinase activity \\
\hline GO:0005010 & 0.004318 & 1 & 3 & Insulin-like growth factor receptor activity \\
\hline GO:0019834 & 0.004318 & 1 & 3 & Phospholipase A2 inhibitor activity \\
\hline GO:0005047 & 0.005753 & 1 & 4 & Signal recognition particle binding \\
\hline GO:0035035 & 0.008618 & 1 & 6 & Histone acetyltransferase binding \\
\hline GO:0008312 & 0.008618 & 1 & 6 & 7S RNA binding \\
\hline GO:0004703 & 0.010048 & 1 & 7 & G-protein coupled receptor kinase activity \\
\hline GO:0031369 & 0.011475 & 1 & 8 & Translation initiation factor binding \\
\hline
\end{tabular}

GO, gene ontology; DEG, differentially expressed genes; RVTE, recurrent venous thromboembolism; SVTE, single venous thromboembolism; Non-overlap, non-overlapping DEGs as specific DEGs of RVTE; cAMP, cyclic adenosine monophosphate; GOMFID, gene ontology molecular function identification number; Count, DEG numbers enriched in the specific GO term; Size, total gene numbers in the GO database.

VTE by successfully reducing morbidity and mortality (21). However, to date, methods for effectively preventing and diagnosing SVTE and RVTE have remained controversial (22). The present study used bioinformatics methods to investigate the molecular mechanisms and potential biomarkers of SVTE and RVTE.

In the present study, gene expression profiles of whole blood samples were successfully used to screen for DEGs in specimens from patients with SVTE compared with those in control specimens. With regard to enriched biological processes and pathways for DEGs in SVTE, genes involved in ribosomal pathways, including RPS3A and RPS7, and mitochondrial function, including UQCRQ and COX7C, were indicated to be most consistently affected and modulated. Ribosomal proteins have remained highly conserved during evolution and reflect critical functions in ribosome biogenesis; in addition, several ribosomal proteins were shown to have extra-ribosomal functions in apoptosis, DNA repair and genetic disease (23). A total of 12 DEGs were involved in ribosomal pathways. A paucity of studies have explored the pathogenesis of VTE. It has previously been indicated that the ribosomal-related RP-MDM2-P53 axis may be involved in the molecular pathogenesis of the $5 q$ syndrome, and VTE was reported in $3 \%$ of patients with $5 q$ syndrome (24). 
Table V. KEGG pathway enrichment analysis of DEGs in patients with RVTE and SVTE, and specific DEGs of RVTE.

\begin{tabular}{|c|c|c|c|c|}
\hline Category/KEGGID & P-value & Count (n) & Size (n) & Term \\
\hline \multicolumn{5}{|l|}{ RVTE } \\
\hline K03010 & $1.59 \times 10^{-23}$ & 17 & 86 & Ribosome \\
\hline K05012 & 0.007377 & 4 & 133 & Parkinson's disease \\
\hline K00190 & 0.007772 & 4 & 135 & Oxidative phosphorylation \\
\hline K05010 & 0.017421 & 4 & 171 & Alzheimer's disease \\
\hline K05016 & 0.022594 & 4 & 185 & Huntington's disease \\
\hline \multicolumn{5}{|l|}{ SVTE } \\
\hline K03010 & $4.58 \times 10^{-19}$ & 12 & 86 & Ribosome \\
\hline K04260 & 0.025106 & 2 & 79 & Cardiac muscle contraction \\
\hline \multicolumn{5}{|l|}{ Non-overlap } \\
\hline K03010 & $2.25 \times 10^{-6}$ & 5 & 86 & Ribosome \\
\hline K03060 & 0.03007 & 1 & 11 & Protein export \\
\hline
\end{tabular}

KEGG, Kyoto Encyclopedia of Genes and Genomes; DEG, differentially expressed genes; RVTE, recurrent venous thromboembolism; SVTE, single venous thromboembolism; Non-overlap, non-overlapping DEGs as specific DEGs of RVTE. KEGGID, KEGG identification number. Count, number of DEGs enriched in the specific GO term; size, number of genes in the GO database.

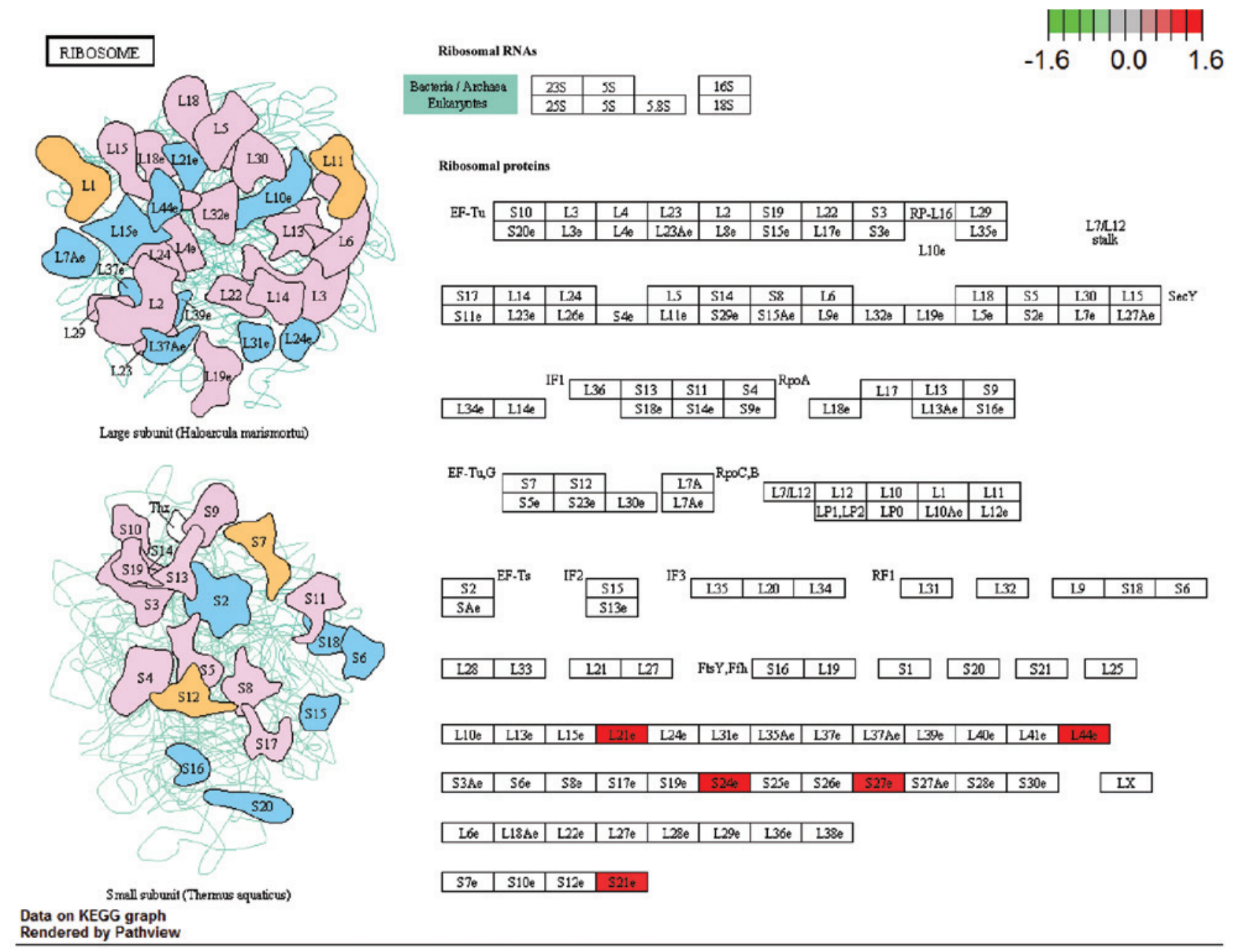

Figure 2. Visualization of the ribosome pathway. Red nodes represent upregulated differentially expressed genes.

COX7C and UQCRQ are constituents of the mitochondrial respiratory chain (25). Mutations of these two genes may increase oxidative stress in coronary artery disease (26). The mortality after VTE is strongly associated with presentation 


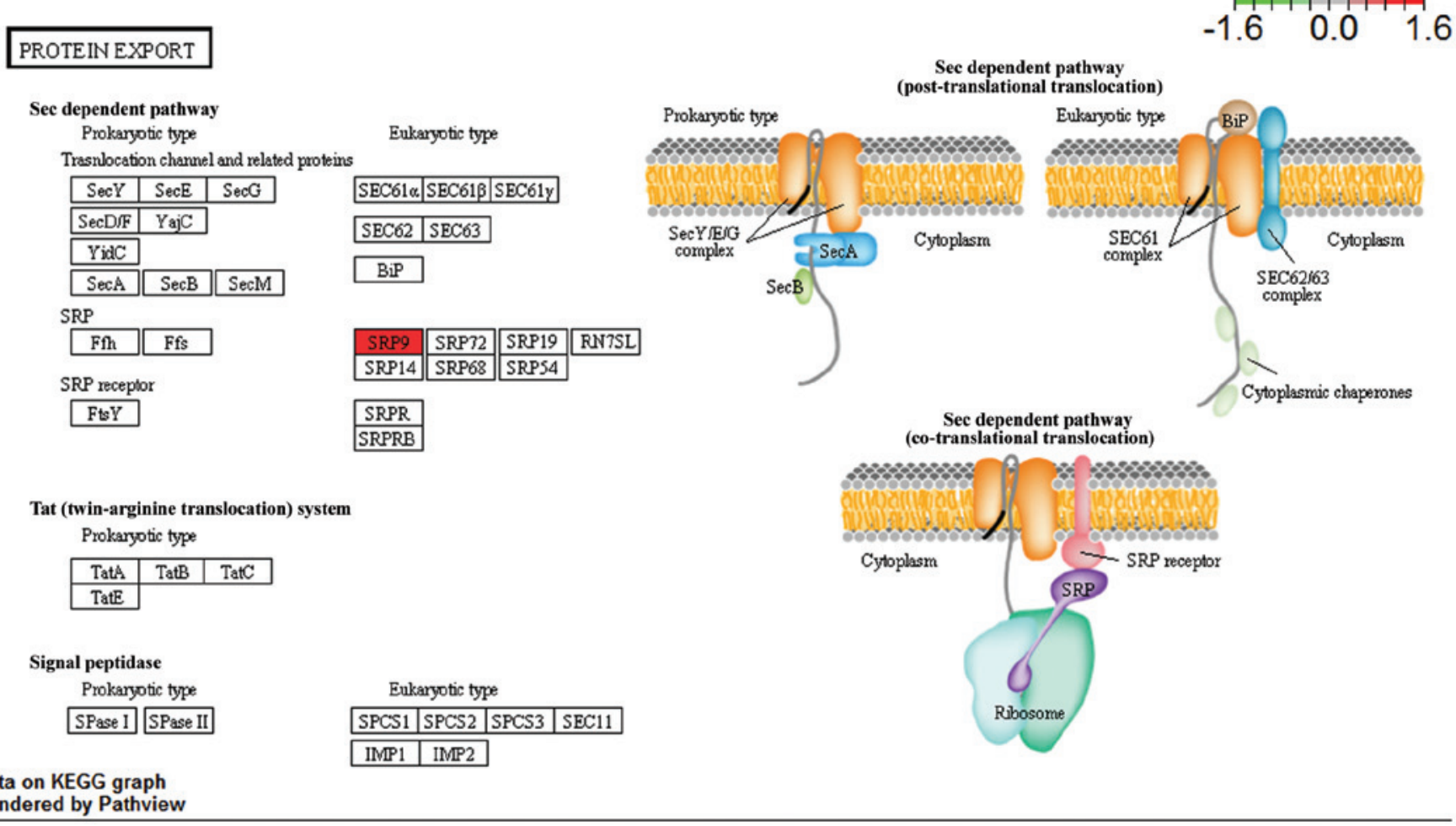

Figure 3. Visualization of the protein export pathway. Red nodes represent upregulated differentially expressed genes.

of underlying cardiovascular disease (27). First-time VTE in numerous patients is idiopathic and challenging to diagnose, while COX7C and UQCRQ may represent novel biomarkers to identify SVTE.

The specific DEGs in RVTE were found to be mainly involved in ribosomal pathways, heart contraction and oxidative phosphorylation. Pathway visualization revealed that RPL21, RPS21, RPS24 and RPS27, which all encode ribosomal proteins, were enriched in ribosomal pathways, while SRP9 was enriched in the protein export pathway. Furthermore, RPL21, RPS21, RPS24 and RPS27 were found to be involved in RVTE through critical ribosome biogenesis or extra-ribosomal functions; of note, the expression of these genes was upregulated in patients with RVTE but unchanged in patients with SVTE. It has been reported that certain diseases, including transient ischemia/reperfusion and pre-eclampsia, are associated with ribosomes $(28,29)$. RPS24 mutation was potentially linked to pathologies of Diamond-Blackfan anemia (30). The above results suggested that RPL21, RPS21, RPS24 and RPS27 may have critical roles in RVTE.

The results of the present study showed that $\beta$-adrenergic receptor kinase 1 (ADRBK1) was closely associated with RVTE and involved in heart contraction. Polymorphisms in ADRB2 and LPL genes are known to have central roles in vascular biology (31). A previous study suggested that the use of ADRBK1 as a biomarker significantly improved heart failure therapy (32). Certain studies showed that the ADRBK1/phosphoinositide-3 kinase(PI3K) complex improved cardiac function and that PI3K-dependent phosphorylation of ADRBK1 on Ser670 is responsible for the downregulation of
ADRBK1 protein via a proteasome-dependent pathway $(33,34)$. Furthermore, PI3K influences insulin-like growth factor and blood vessel-related factor through $\mathrm{G}$ protein beta gamma (35). These results may suggest that ADRBK1 has a critical role in RVTE by serving as a dual effector of the compensatory myocardial diastole and the PI3K response.

The present study also identified NDUFA5 and ATP ATP5O as DEGs, which were significantly associated with oxidative phosphorylation. The oxidative stress injury and exitotoxicity in mitochondria induced by NDUFA5 and ATP5O have been proved to be the cause of a variety of nervous system degenerative diseases, including Parkinson's, Alzheimer's and Huntington's disease $(36,37)$. Free-radical generation and consequent oxidative stress in platelet activation and thrombotic vascular diseases have a distinctive role with the potential injurious effects of homocysteine $(38,39)$. Therefore, NDUFA5 and ATP5O inducing oxidative stress injury and exitotoxicity in mitochondria may also have an impact on RVTE.

In conclusion, the screening performed in the present study identified 42 DEGs in RVTE, including 35 up- and 7 downregulated genes, 20 DEGs in SVTE, including 17 up- and 3 downregulated genes, and 22 specific DEGs in RVTE. Furthermore, functional and pathway enrichment analysis was performed for these identified DEGs. The results indicated that DEGs in SVTE, including COX7C and UQCRQ, may be used as potential biomarkers for SVTE and that specific DEGs in RVTE, including ADRBK1, NDUFA5 and ATP5O, may be considered as potential biomarkers of RVTE. However, experimental studies are required to confirm these results. 


\section{Acknowledgments}

The present study was supported by the National Natural Science Foundation of China (grant no. 81370255).

\section{References}

1. Pabinger I and Ay C: Biomarkers and venous thromboembolism Arterioscler Thromb Vasc Biol 29: 332-336, 2009.

2. Baglin T: What happens after venous thromboembolism? J Thromb Haemost 7 (Suppl 1): 287-290, 2009.

3. Stein PD, Beemath A and Olson RE: Trends in the incidence of pulmonary embolism and deep venous thrombosis in hospitalized patients. Am J Cardiol 95: 1525-1526, 2005.

4. Nielsen JD: The incidence of pulmonary embolism during deep vein thrombosis. Phlebology 28 (Suppl 1): S29-S33, 2013.

5. Heit JA, Mohr DN, Silverstein MD, Petterson TM, O'Fallon WM and Melton LJ III: Predictors of recurrence after deep vein thrombosis and pulmonary embolism: A population-based cohort study. Arch Intern Med 160: 761-768, 2000.

6. Poli D and Palareti G: Assessing recurrence risk following acute venous thromboembolism: Use of algorithms. Curr Opin Pulm Med 19: 407-412, 2013.

7. Barnes DM, Wakefield TW and Rectenwald JE: Novel biomarkers associated with deep venous thrombosis: A comprehensive review. Biomarker Insights 3: 93-100, 2008.

8. Kyrle PA, Minar E, Hirschl M, Bialonczyk C, Stain M, Schneider B, Weltermann A, Speiser W, Lechner K and Eichinger S: High plasma levels of factor VIII and the risk of recurrent venous thromboembolism. N Engl J Med 343: 457-462, 2000.

9. Comp PC and Esmon CT: Recurrent venous thromboembolism in patients with a partial deficiency of protein S. N Engl J Med 311: $1525-1528,1984$

10. Palareti G, Cosmi B, Legnani C, Tosetto A, Brusi C, Iorio A, Pengo V, Ghirarduzzi A, Pattacini C, Testa S, et al: D-dimer testing to determine the duration of anticoagulation therapy. $\mathrm{N}$ Engl J Med 355: 1780-1789, 2006.

11. Pabinger I, Thaler J and Ay C: Biomarkers for prediction of venous thromboembolism in cancer. Blood 122: 2011-2018, 2013.

12. Ay C, Dunkler D, Simanek R, Thaler J, Koder S, Marosi C, Zielinski $\mathrm{C}$ and Pabinger I: Prediction of venous thromboembolism in patients with cancer by measuring thrombin generation: Results from the Vienna cancer and thrombosis study. J Clin Oncol 29: 2099-2103, 2011.

13. Bouman AC, Smits JJ, Ten Cate $H$ and Ten Cate-Hoek AJ: Markers of coagulation, fibrinolysis and inflammation in relation to post-thrombotic syndrome. J Thromb Haemost 10: 1532-1538, 2012

14. Lewis DA, Stashenko GJ, Akay OM, Price LI, Owzar K Ginsburg GS, Chi JT and Ortel TL: Whole blood gene expression analyses in patients with single vs. recurrent venous thromboembolism. Thromb Res 128: 536-540, 2011.

15. Gentleman RC, Carey VJ, Bates DM, Bolstad B, Dettling M, Dudoit S, Ellis B, Gautier L, Ge Y, Gentry J, et al: Bioconductor: open software development for computational biology and bioinformatics. Genome Biol 5: R80, 2004.

16. Irizarry RA, Bolstad BM, Collin F, Cope LM, Hobbs B and Speed TP: Summaries of affymetrix genechip probe level data. Nucleic Acids Res 31: e15, 2003.

17. Wilson CL and Miller CJ: Simpleaffy: A Bioconductor package for affymetrix quality control and data analysis. Bioinformatics 21: 3683-3685, 2005 .

18. R Development Core Team: R: A Language and Environment for Statistical Computing. The R Foundation for Statistical Computing, Vienna, Austria, 2011. ISBN: 3-900051-07-0.

19. Zheng G, Wang H, Wei C and Li Y: iGepros: An integrated gene and protein annotation server for biological nature exploration. BMC Bioinformatics 12 (Suppl 14): S6, 2011.

20. Luo W and Brouwer C: Pathview: an R/Bioconductor package for pathway-based data integration and visualization. Bioinformatics 29: 1830-1831, 2013.
21. Verhamme $\mathrm{P}$ and Bounameaux H: Direct oral anticoagulants for acute venous thromboembolism: Closing the circle? Circulation 129: 725-727, 2014.

22. Lippi G, Cervellin G, Franchini M and Favaloro EJ: Biochemical markers for the diagnosis of venous thromboembolism: The past, present and future. J Thromb Thrombolysis 30: 459-471, 2010.

23. Lindström MS: Emerging functions of ribosomal proteins in gene-specific transcription and translation. Biochem Biophys Res Commun 379: 167-170, 2009.

24. Komrokji RS, Padron E, Ebert BL and List AF: Deletion 5q MDS: Molecular and therapeutic implications. Best Pract Res Clin Haematol 26: 365-375, 2013.

25. Beech RD, Lowthert L, Leffert JJ, Mason PN, Taylor MM, Umlauf S, Lin A, Lee JY, Maloney K, Muralidharan A, et al: Increased peripheral blood expression of electron transport chain genes in bipolar depression. Bipolar disorders 12: 813-824, 2010.

26. Taurino C, Miller WH, McBride MW, McClure JD, Khanin R, Moreno MU, Dymott JA, Delles C and Dominiczak AF: Gene expression profiling in whole blood of patients with coronary artery disease. Clin Sci (Lond) 119: 335-343, 2010.

27. White RH: The epidemiology of venous thromboembolism Circulation 107: I4-I8, 2003.

28. Textoris J, Ivorra D, Ben Amara A, Sabatier F, Ménard JP, Heckenroth H, Bretelle F and Mege JL: Evaluation of current and new biomarkers in severe preeclampsia: A microarray approach reveals thevs.ig4 gene as a potential blood biomarker. PLoS One 8: e82638, 2013

29. White BC, Sullivan JM, DeGracia DJ, O'Neil BJ, Neumar RW, Grossman LI, Rafols JA and Krause GS: Brain ischemia and reperfusion: Molecular mechanisms of neuronal injury. J Neurol Sci 179: 1-33, 2000.

30. Choesmel V,Fribourg S, Aguissa-Touré AH, Pinaud N, Legrand P, Gazda HT and Gleizes PE: Mutation of ribosomal protein RPS24 in Diamond-Blackfan anemia results in a ribosome biogenesis disorder. Hum Mol Genet 17: 1253-1263, 2008.

31. Zee RY, Cook NR, Cheng S, Erlich HA, Lindpaintner K and Ridker PM: Polymorphism in the beta2-adrenergic receptor and lipoprotein lipase genes as risk determinants for idiopathic venous thromboembolism: A multilocus, population-based, prospective genetic analysis. Circulation 113: 2193-2200, 2006.

32. Tachibana H, Naga Prasad SV, Lefkowitz RJ, Koch WJ and Rockman HA: Level of beta-adrenergic receptor kinase 1 inhibition determines degree of cardiac dysfunction after chronic pressure overload-induced heart failure. Circulation 111: 591-597, 2005.

33. Ito K, Akazawa H, Tamagawa M, Furukawa K, Ogawa W, Yasuda N, Kudo Y, Liao CH, Yamamoto R, Sato T, et al: PDK1 coordinates survival pathways and beta-adrenergic response in the heart. Proc Natl Acad Sci USA 106: 8689-8694, 2009.

34. Lombardi MS, Vroon A, Sodaar P, van Muiswinkel FL, Heijnen CJ and Kavelaars A: Down-regulation of GRK2 after oxygen and glucose deprivation in rat hippocampal slices: Role of the PI3-kinase pathway. J Neurochem 102: 731-740, 2007.

35. Hideshima T, Nakamura N, Chauhan D and Anderson KC Biologic sequelae of interleukin-6 induced PI3-K/Akt signaling in multiple myeloma. Oncogene 20: 5991-6000, 2001.

36. Chou JL, Shenoy DV, Thomas N, Choudhary PK, Laferla FM, Goodman SR and Breen GA: Early dysregulation of the mitochondrial proteome in a mouse model of Alzheimer's disease. J Proteomics 74: 466-479, 2011.

37. Weydt P, Pineda VV, Torrence AE, Libby RT, Satterfield TF, Lazarowski ER, Gilbert ML, Morton GJ, Bammler TK, Strand AD, et al: Thermoregulatory and metabolic defects in Huntington's disease transgenic mice implicate PGC-1alpha in Huntington's disease neurodegeneration. Cell Metab 4: 349-362, 2006.

38. Davi G, Di Minno G, Coppola A, Andria G, Cerbone AM, Madonna P, Tufano A, Falco A, Marchesani P, Ciabattoni G and Patrono C: Oxidative stress and platelet activation in homozygous homocystinuria. Circulation 104: 1124-1128, 2001.

39. El Kossi MM and Zakhary MM: Oxidative stress in the context of acute cerebrovascular stroke. Stroke 31: 1889-1892, 2000. 